\title{
4-WEEK INHALATION TOXICITY OF 2-METHYLNAPHTHALENE IN EXPERIMENTAL ANIMALS
}

\section{RADOSŁAW ŚWIERCZ, WOJCIECH WĄSOWICZ, JAN STETKIEWICZ, JOLANTA GROMADZIŃSKA, and WANDA MAJCHEREK}

Nofer Institute of Occupational Medicine, Łódź, Poland

Department of Toxicology and Carcinogenesis

\begin{abstract}
Objectives: This paper presents toxic effects of 2-MN in laboratory animals under conditions of 4-week inhalation exposure to 2-methylnaphthalene (2-MN) vapors. Materials and Methods: Male Wistar rats were exposed to 2-MN vapors at a nominal concentration of $0,2,10$ or $50 \mathrm{mg} / \mathrm{m}^{3}$ in dynamic inhalation chambers for 4 weeks $(6 \mathrm{~h} /$ day, 5 days/week). After 4 weeks of inhalation exposure the animals were necropsied. Blood samples were collected and selected organs were weighted and prepared for histological examinations. Results: The effects of the increased levels of exposure to 2-MN experienced by the experimental rats were as follows: a) increasing $\gamma$-glutamylotransferase activity, b) stimulation of the hematopoietic system, c) lower cholesterol concentrations, d) higher number of goblet cells in lobar bronchi, e) hyperplasia of hepatic bile ducts. Conclusion: Four-week exposure of the animals to $2-\mathrm{MN}$ at $2 \mathrm{mg} / \mathrm{m}^{3}$ proved to be the no-observed-adverse-effect-level (NOAEL), while $10 \mathrm{mg} / \mathrm{m}^{3}$ appeared to represent the lowest-observed-adverseeffect-level (LOAEL).
\end{abstract}

Key words:

2-Methylnaphthalene, Rats, Inhalation, NOAEL, LOAEL

\section{INTRODUCTION}

2-Methylnaphthalene (2-MN) is a natural component of crude oil and coal, and is found in pyrolysis and combustion products such as cigarette and wood smoke, emissions from combustion engines, asphalt, coal tar residues, and used oils. No epidemiology studies or case reports are available which examine the potential effects of human exposure to 2-MN, and no studies are available in which health effects were evaluated in animals following the prechronic or chronic inhalation exposure to 2-MN [1].
Occupational exposure to 2-MN is associated with the process of its manufacture, leather tanning, wood impregnation, crude oil refining, production of dyes, vitamin $\mathrm{K}$ and carbaryl insecticide. The total number of workers exposed in Poland to 2-MN is estimated to equal several hundred people. The current Polish 2-MN maximum allowable concentration (MAC) values for time-weighted average (TWA) and short-term exposure limit (STEL) are 25 and $50 \mathrm{mg} / \mathrm{m}^{3}$, respectively [2].

This paper reports 2-MN toxic activity towards laboratory animals under the conditions of a 4-week inhalation

Received: May 4, 2011. Accepted: July 11, 2011.

Address reprint requests to R. Świercz, Department of Toxicology and Carcinogenesis, Nofer Institute of Occupational Medicine, św. Teresy 8, 91-348 Łódź, Poland (e-mail: radek@imp.lodz.pl). 
exposure to 2-MN vapors at the target concentration of $0,2,10$ and $50 \mathrm{mg} / \mathrm{m}^{3}$.

\section{MATERIALS AND METHODS}

\section{Chemicals}

2-Methylnaphthalene (2-MN, No. CAS: 91-57-6) was supplied by Fluka; its purity was $97 \%$. The conversion factors for 2-MN: $1 \mathrm{ppm} \sim 5.81 \mathrm{mg} / \mathrm{m}^{3}$.

\section{Animals and inhalation exposure}

Female and male Wistar rats IMP: WIST (five animals in each group) were exposed to 2-MN vapors at the target concentration of $0,2,10$ and $50 \mathrm{mg} / \mathrm{m}^{3}$ in dynamic inhalation chambers (volume $0.25 \mathrm{~m}^{3}, 15$ air changes per hour) for 4 weeks ( $6 \mathrm{~h} /$ day, 5 days/week). Body weights and food consumption were measured weekly. The animals were given standard laboratory food and water ad libitum, except for the time during which they were exposed to 2-MN vapors in dynamic inhalation chambers. The chamber relative temperature and humidity were maintained at $22-24^{\circ} \mathrm{C}$ and $35-45 \%$, respectively. The required $2-\mathrm{MN}$ vapors were generated by heating 2-MN to $85^{\circ} \mathrm{C}$ in a glass washer. The desired concentrations of vapors were obtained by diluting them with air. A vapor sample of 2-methylnaphtalene $\left(0.5 \mathrm{dm}^{3}\right)$ was absorbed on $2 \mathrm{~cm}^{3}$ liquid sorbent (ethyl alcohol from Polmos, purity 95\%). The concentration of solvent vapors in the exposure chamber was measured every $30 \mathrm{~min}$ by gas chromatography (Hewlett-Packard 5890) with a flame ionization detector (FID) using capillary column (HP-1; 5 m, 0.53 mm, $2.65 \mu \mathrm{m}$ film thickness). The operating conditions were: carrier gas - helium, column flow $10 \mathrm{ml} / \mathrm{min}$; make-up gas (helium) $20 \mathrm{ml} / \mathrm{min}$; air $300 \mathrm{ml} / \mathrm{min}$; oven $150^{\circ} \mathrm{C}$; inlet split $220^{\circ} \mathrm{C}$, detector $230^{\circ} \mathrm{C}$.

\section{Biological material collection and analysis}

Blood and organs of rats were collected after the 4-week inhalation exposure to 2-MN. The animals were anesthetized in light ether anesthesia, exsanguinated from the femoral artery and vein, decapitated and subjected to gross necropsy.

The necropsy was performed in all animals. Organ weights were obtained for lungs, liver, spleen, kidneys, adrenals, heart and gonads (ovaries, testes). To facilitate histopathological investigation, brain, nose, larynx, trachea, thymus, lungs, heart, liver, spleen, kidney, adrenals, thyroid gland, pancreas, gonads, urinary bladder, stomach, duodenum, small and large intestines, and salivary glands were preserved in neutral formalin.

Erythrocyte count, hemoglobin concentration, hematocrit value, leukocyte count, platelet count and clotting time were determined in the control and exposure group of animals.

Blood samples were collected for serum biochemistry determinations. Alanine aminotranferase, alkaline phosphatase, aspartate aminotranferase, total protein, total cholesterol, $\gamma$-glutamyltransferase, glucose, creatinine, urea, sorbitol dehydrogenase, albumin, sodium, potassium and chloride were determined. Beckman Clinical System 700 autoanalyser, Ciba-Corning 614 analyser and standard test combination kits were used for the determinations.

Lungs with larynx and trachea were removed in one piece and fixed with buffered $10 \%$ formalin solution by instillation at $20 \mathrm{~cm}$ water pressure. The soft tissues were trimmed and embedded in paraffin. They were sectioned at $5 \mu \mathrm{m}$ and stained with hematoxylin and eosin. Sections from trachea and lungs were additionally stained with periodic acid Schiff's reagent. The liver and kidney frozen sections were stained with Fat Red 7B stain.

\section{Statistical analysis}

Differences were assessed using the Kruskall-Wallis test in Dunn's modification [3]. The trend analysis was performed with the use of the Jonckheere's test [4]. The differences were regarded as significant when the probability of the null hypothesis was $<0.05$. 


\section{RESULTS}

All the rats survived inhalation exposure to 2-MN. Table 1 gives target and actual 2-MN concentrations in toxicological chambers and the mean values of body mass and food intake of exposed animals during the 4-week exposure. Compared with the controls, no statistically significant changes were noted in body mass or food intake in the case of the majority of exposed animals during the 4-week exposure. The only significant reduction of body mass was noted on measurement day 14 in the male rats exposed to 2-MN at $10 \mathrm{mg} / \mathrm{m}^{3}$. Reduced food intakes and lower body weights were recorded on measurement days 21 and 28 in female rats exposed to 2-MN at 10 and $50 \mathrm{mg} / \mathrm{m}^{3}$ vs. the controls. Macroscopic examinations did not reveal any significant changes between the controls and the exposed animals. Table 2 shows the mass of the internal organs of the studied animals at the end of the 4-week inhalation exposure to 2-MN. A significant reduction of absolute and relative liver mass was recorded in all male rats exposed to various 2-MN concentrations compared to the control group. All exposed male rats were noted to show a tendency to slightly reduced absolute mass of spleen compared to the control group, while the reduction was significant in the group exposed to $2-\mathrm{MN}$ at $10 \mathrm{mg} / \mathrm{m}^{3}$. A significant increase in the relative mass of lungs was noted in the group of male animals exposed to $10 \mathrm{mg} / \mathrm{m}^{3}$ 2-MN compared to the controls. The female rats were found to display a 2-MN-concentration-dependent tendency to reduced absolute mass of lungs, liver, spleen, kidneys and heart, and smaller relative mass of spleen. The relative and absolute mass of the ovaries was found to be significantly greater, compared to the control group, in the group of female rats exposed to $2 \mathrm{mg} / \mathrm{m}^{3} 2-\mathrm{MN}$.

Table 3 specifies hematological data of the controls and 2-MN-exposed rats. A significant, 2-MN-concentration-dependent increase in hematocrit value and reticulocyte percentage, higher hemoglobin concentration and erythrocyte and leukocyte counts were noted in the male rats, while the females showed a 2-MN-concentration-dependent increase in the proportion of reticulocytes.

Table 4 shows the results of biochemical tests of blood serum from control and 2-MN-exposed rats. With growing 2-MN exposure, statistically significant increase in alkaline phosphatase and $\gamma$-glutamyltransferase activity, lower sorbitol dehydrogenase activity and lower albumin concentration were noted in the serum of exposed rats. The total cholesterol concentration in the serum of 2-MN-exposed male rats was significantly higher in the $2 \mathrm{mg} / \mathrm{m}^{3}$ group, while in the remaining 2-MN-exposed groups, lower values were observed. Compared to the controls, lower alanine aminotransferase activity and lower glucose, urea and sodium concentrations were noted in the serum of male rats exposed to $2-\mathrm{MN}$ at $2 \mathrm{mg} / \mathrm{m}^{3}$, there was also a significant reduction of glucose concentration in the group exposed to 2-MN at $10 \mathrm{mg} / \mathrm{m}^{3}$. In the serum of the female rats, there was a significant 2-MN concentration-dependent increase of glucose, urea and sodium concentrations and a reduction of total cholesterol concentration, while the group of females exposed to $2-\mathrm{MN}$ at $2 \mathrm{mg} / \mathrm{m}^{3}$ experienced a significant reduction of albumin concentrations.

Pathological changes were not found in controls of both genders during pathomorphological examinations of the respiratory system. The microscopic structure of the lungs was normal. Cylindrical epithelium of primary and lobar bronchi was well preserved. Single goblet cells could be observed solely in the epithelium of the primary bronchi. Peribronchial lymphatic tissue was meagre.

Single goblet cells in the epithelium of the primary and lobar bronchi of the animals exposed to 2-MN at $\mathrm{mg} / \mathrm{m}^{3}$ were observed in two (2/5) males and three (3/5) females. Moderate hyperplasia of the peribronchial lymphatic tissue was noted in one $(1 / 5)$ male rat.

Elevated numbers of goblet cells were noted in the primary and lobar bronchi of four male (4/5) and three female (3/5) rats exposed to 2-MN at $10 \mathrm{mg} / \mathrm{m}^{3}$. Exposure to 2-MN 
Table 1. Mean air concentrations of 2-MN in inhalation chambers and body weight and food intake in rats subjected to a repeated exposure to 2-MN

\begin{tabular}{|c|c|c|c|c|c|c|c|}
\hline \multicolumn{2}{|c|}{$\begin{array}{l}\text { 2-MN concentration } \\
\left(\mathrm{mg} / \mathrm{m}^{3} \pm \mathrm{SD}\right)\end{array}$} & \multirow[t]{2}{*}{ Parameter } & \multicolumn{5}{|c|}{ Day } \\
\hline target air & inhaled air & & 1 & 7 & 14 & 21 & 28 \\
\hline \multirow[t]{6}{*}{ Controls } & 0 & $\begin{array}{l}\text { Body weight } \\
(\mathrm{g} \pm \mathrm{SD})\end{array}$ & & & & & \\
\hline & & male & $351.0 \pm 24.3$ & $362.0 \pm 26.8$ & $388.0 \pm 28.6$ & $398.0 \pm 26.1$ & $393.0 \pm 36.0$ \\
\hline & & female & $213.0 \pm 11.5$ & $212.0 \pm 13.0$ & $222.0 \pm 21.1$ & $232.0 \pm 19.2$ & $242.0 \pm 18.2$ \\
\hline & & $\begin{array}{l}\text { Food intake } \\
(\mathrm{g} / \mathrm{rat} / \text { week })\end{array}$ & & & & & \\
\hline & & male & - & 154 & 158 & 173 & 167 \\
\hline & & female & - & 103 & 98 & 120 & 123 \\
\hline \multirow[t]{6}{*}{2} & $2.0 \pm 0.2$ & $\begin{array}{l}\text { Body weight } \\
(\mathrm{g} \pm \mathrm{SD})\end{array}$ & & & & & \\
\hline & & male & $344.0 \pm 9.6$ & $360.0 \pm 15.8$ & $376.0 \pm 17.1$ & $400.0 \pm 12.7$ & $411.0 \pm 16.4$ \\
\hline & & female & $203.0 \pm 2.7$ & $208.0 \pm 8.4$ & $220.0 \pm 13.7$ & $224.0 \pm 10.8$ & $225.0 \pm 11.7$ \\
\hline & & $\begin{array}{l}\text { Food intake } \\
\text { (g/rat/week) }\end{array}$ & & & & & \\
\hline & & male & - & 170 & 157 & 167 & 171 \\
\hline & & female & - & 106 & 109 & 107 & 120 \\
\hline \multirow[t]{6}{*}{10} & $11.0 \pm 1.5$ & $\begin{array}{l}\text { Body weight } \\
(\mathrm{g} \pm \mathrm{SD})\end{array}$ & & & & & \\
\hline & & male & $315.0 \pm 23.2$ & $334.0 \pm 24.1$ & $341.0 \pm 31.3^{*}$ & $367.0 \pm 28.6$ & $379.0 \pm 33.8$ \\
\hline & & female & $199.0 \pm 8.2$ & $204.0 \pm 8.9$ & $216.0 \pm 9.6$ & $217.0 \pm 10.4$ & $222.0 \pm 13.0$ \\
\hline & & $\begin{array}{l}\text { Food intake } \\
\text { (g/rat/week) }\end{array}$ & & & & & \\
\hline & & male & - & 158 & 160 & 168 & 171 \\
\hline & & female & - & 103 & 102 & 108 & 106 \\
\hline \multirow[t]{6}{*}{50} & $51.0 \pm 3.7$ & $\begin{array}{l}\text { Body weight } \\
(\mathrm{g} \pm \mathrm{SD})\end{array}$ & & & & & \\
\hline & & male & $356.0 \pm 18.5$ & $358.0 \pm 23.9$ & $369.0 \pm 19.8$ & $387.0 \pm 18.6$ & $420.0 \pm 40.9$ \\
\hline & & female & $224.0 \pm 23.4$ & $226.0 \pm 26.1$ & $235.0 \pm 28.5$ & $239.0 \pm 28.4$ & $239.0 \pm 24.9$ \\
\hline & & $\begin{array}{l}\text { Food intake } \\
\text { (g/rat/week) }\end{array}$ & & & & & \\
\hline & & male & - & 150 & 155 & 143 & 170 \\
\hline & & female & - & 106 & 103 & 111 & 98 \\
\hline
\end{tabular}

Mean values \pm SD for 5 rats.

* Significantly different from the control group at $\mathrm{p} \leq 0.05$. 
Table 2. Mean values ( $\mathrm{g} \pm \mathrm{SD}$ ) of organs obtained from rats exposed to 2-MN for 4 weeks

\begin{tabular}{|c|c|c|c|c|c|}
\hline \multirow{2}{*}{ Parameters } & \multirow{2}{*}{ Controls } & \multicolumn{3}{|c|}{ 2-MN target concentration in inhaled air $\left(\mathrm{mg} / \mathrm{m}^{3}\right)$} & \multirow{2}{*}{$\begin{array}{c}\text { Jonckheere's trend } \\
\text { test }\left(\geq p_{\mathrm{J}}\right)\end{array}$} \\
\hline & & 2 & 10 & 50 & \\
\hline \multicolumn{6}{|l|}{ Males } \\
\hline \multicolumn{6}{|l|}{$\begin{array}{l}\text { Absolute organ } \\
\text { weight }(\mathrm{g})\end{array}$} \\
\hline lungs & $1.887 \pm 0.213$ & $1.705 \pm 0.114$ & $2.078 \pm 0.430$ & $1.792 \pm 0.143$ & ns \\
\hline liver & $11.981 \pm 1.078$ & $8.821 \pm 0.516^{* *}$ & $9.112 \pm 1.200^{*}$ & $9.433 \pm 0.711^{*}$ & 0.050 \\
\hline spleen & $0.801 \pm 0.074$ & $0.690 \pm 0.094$ & $0.635 \pm 0.072^{*}$ & $0.745 \pm 0.119$ & ns \\
\hline kidneys & $2.395 \pm 0.125$ & $2.206 \pm 0.146$ & $2.096 \pm 0.259$ & $2.422 \pm 0.167$ & ns \\
\hline adrenals & $0.049 \pm 0.006$ & $0.059 \pm 0.025$ & $0.046 \pm 0.007$ & $0.057 \pm 0.025$ & ns \\
\hline heart & $0.979 \pm 0.135$ & $0.934 \pm 0.027$ & $0.937 \pm 0.068$ & $0.991 \pm 0.113$ & ns \\
\hline testes & $3.567 \pm 0.281$ & $3.331 \pm 0.444$ & $3.521 \pm 0.357$ & $3.761 \pm 0.417$ & $\mathrm{~ns}$ \\
\hline \multicolumn{6}{|c|}{$\begin{array}{l}\text { Relative organ } \\
\text { weight ( } \mathrm{g} / 100 \mathrm{~g} \text { b.w.) }\end{array}$} \\
\hline lungs & $0.458 \pm 0.030$ & $0.442 \pm 0.017$ & $0.596 \pm 0.185^{*}$ & $0.450 \pm 0.051$ & ns \\
\hline liver & $2.912 \pm 0.210$ & $2.286 \pm 0.098^{* * *}$ & $2.554 \pm 0.149$ & $2.375 \pm 0.312^{*}$ & 0.050 \\
\hline spleen & $0.195 \pm 0.014$ & $0.179 \pm 0.022$ & $0.181 \pm 0.034$ & $0.189 \pm 0.042$ & ns \\
\hline kidneys & $0.583 \pm 0.027$ & $0.573 \pm 0.058$ & $0.589 \pm 0.046$ & $0.608 \pm 0.056$ & ns \\
\hline adrenals & $0.012 \pm 0.002$ & $0.015 \pm 0.006$ & $0.013 \pm 0.003$ & $0.015 \pm 0.006$ & ns \\
\hline heart & $0.237 \pm 0.017$ & $0.242 \pm 0.012$ & $0.264 \pm 0.019$ & $0.249 \pm 0.040$ & ns \\
\hline testes & $0.867 \pm 0.061$ & $0.862 \pm 0.089$ & $0.999 \pm 0.162$ & $0.953 \pm 0.177$ & ns \\
\hline \multicolumn{6}{|l|}{ Females } \\
\hline \multicolumn{6}{|l|}{$\begin{array}{l}\text { Absolute organ } \\
\text { weight }(\mathrm{g})\end{array}$} \\
\hline lungs & $1.472 \pm 0.238$ & $1.457 \pm 0.326$ & $1.292 \pm 0.090$ & $1.257 \pm 0.130$ & 0.050 \\
\hline liver & $6.855 \pm 1.076$ & $6.072 \pm 0.486$ & $5.643 \pm 0.610$ & $5.377 \pm 0.572^{*}$ & 0.005 \\
\hline spleen & $0.493 \pm 0.074$ & $0.644 \pm 0.083$ & $0.512 \pm 0.056$ & $0.367 \pm 0.053$ & 0.020 \\
\hline kidneys & $1.556 \pm 0.091$ & $1.418 \pm 0.049$ & $1.328 \pm 0.067^{* *}$ & $1.406 \pm 0.081^{*}$ & 0.010 \\
\hline adrenals & $0.071 \pm 0.009$ & $0.074 \pm 0.013$ & $0.063 \pm 0.013$ & $0.063 \pm 0.007$ & $\mathrm{~ns}$ \\
\hline heart & $0.724 \pm 0.050$ & $0.656 \pm 0.031$ & $0.645 \pm 0.046$ & $0.568 \pm 0.073^{* *}$ & 0.005 \\
\hline ovaries & $0.074 \pm 0.004$ & $0.125 \pm 0.021^{*}$ & $0.077 \pm 0.015$ & $0.071 \pm 0.010$ & $\mathrm{~ns}$ \\
\hline \multicolumn{6}{|c|}{$\begin{array}{l}\text { Relative organ } \\
\text { weight ( } \mathrm{g} / 100 \mathrm{~g} \text { b.w.) }\end{array}$} \\
\hline lungs & $0.620 \pm 0.063$ & $0.680 \pm 0.141$ & $0.627 \pm 0.028$ & $0.685 \pm 0.076$ & $\mathrm{~ns}$ \\
\hline liver & $2.943 \pm 0.363$ & $2.836 \pm 0.164$ & $2.734 \pm 0.198$ & $2.923 \pm 0.249$ & $\mathrm{~ns}$ \\
\hline spleen & $0.212 \pm 0.031$ & $0.301 \pm 0.031^{* *}$ & $0.248 \pm 0.021$ & $0.199 \pm 0.019$ & 0.050 \\
\hline kidneys & $0.670 \pm 0.039$ & $0.663 \pm 0.008$ & $0.645 \pm 0.023$ & $0.765 \pm 0.018$ & ns \\
\hline adrenals & $0.030 \pm 0.003$ & $0.035 \pm 0.007$ & $0.030 \pm 0.005$ & $0.034 \pm 0.004$ & $\mathrm{~ns}$ \\
\hline heart & $0.313 \pm 0.027$ & $0.307 \pm 0.014$ & $0.314 \pm 0.027$ & $0.310 \pm 0.049$ & ns \\
\hline ovaries & $0.032 \pm 0.003$ & $0.058 \pm 0.008^{* *}$ & $0.038 \pm 0.006$ & $0.039 \pm 0.005$ & $\mathrm{~ns}$ \\
\hline
\end{tabular}

Mean values \pm SD for 5 rats.

$*, * *, * *$ Significantly different from the control group at $\mathrm{p} \leq 0.05, \mathrm{p} \leq 0.01$ and $\mathrm{p} \leq 0.001$, respectively.

ns - not significant. 
Table 3. Hematological results obtained in rats exposed to 2-MN for 4 weeks

\begin{tabular}{|c|c|c|c|c|c|}
\hline \multirow{2}{*}{ Parameters } & \multirow{2}{*}{ Controls } & \multicolumn{3}{|c|}{ 2-MN target concentration in inhaled air $\left(\mathrm{mg} / \mathrm{m}^{3}\right)$} & \multirow{2}{*}{$\begin{array}{c}\text { Jonckheere's } \\
\text { trend test } \\
\left(\geq \mathrm{p}_{\mathrm{J}}\right)\end{array}$} \\
\hline & & 2 & 10 & 50 & \\
\hline \multicolumn{6}{|l|}{ Males } \\
\hline hematocrit $(\%)$ & $43.60 \pm 0.96$ & $42.40 \pm 2.92$ & $45.50 \pm 1.80$ & $45.20 \pm 1.53$ & 0.020 \\
\hline hemoglobin (g/dl) & $14.60 \pm 0.36$ & $14.40 \pm 1.12$ & $13.10 \pm 6.28$ & $15.70 \pm 0.31^{*}$ & 0.010 \\
\hline red blood cells $\left(\times 10^{6} / \mathrm{mm}^{3}\right)$ & $8.42 \pm 0.19$ & $8.37 \pm 0.58$ & $9.58 \pm 0.53^{*}$ & $9.04 \pm 0.57$ & 0.010 \\
\hline white blood cells $\left(\times 10^{3} / \mathrm{mm}^{3}\right)$ & $8.18 \pm 3.48$ & $9.67 \pm 5.27$ & $8.16 \pm 1.46$ & $8.54 \pm 2.01$ & 0.050 \\
\hline \multicolumn{6}{|l|}{ Differential variable $\left(\times 10^{3} / \mathrm{mm}^{3}\right)$} \\
\hline rod neutrophil & $0.0 \pm 0.0$ & $0.03 \pm 0.03$ & $0.01 \pm 0.03$ & $0.0 \pm 0.0$ & ns \\
\hline segmented neutrophil & $1.44 \pm 0.93$ & $2.13 \pm 1.62$ & $1.45 \pm 0.43$ & $1.60 \pm 0.72$ & ns \\
\hline eosinophil & $0.41 \pm 0.20$ & $0.72 \pm 0.78$ & $0.63 \pm 0.19$ & $0.45 \pm 0.26$ & $\mathrm{~ns}$ \\
\hline basophil & $0.0 \pm 0.0$ & $0.0 \pm 0.0$ & $0.0 \pm 0.0$ & $0.0 \pm 0.0$ & ns \\
\hline limphocyte & $6.08 \pm 2.43$ & $6.48 \pm 2.79$ & $5.77 \pm 1.19$ & $6.24 \pm 1.59$ & ns \\
\hline monocyte & $0.24 \pm 0.14$ & $0.31 \pm 0.28$ & $0.28 \pm 0.15$ & $0.25 \pm 0.19$ & ns \\
\hline blood platelet $\left(\times 10^{3} / \mathrm{mm}^{3}\right)$ & $724.30 \pm 84.60$ & $730.50 \pm 90.50$ & $803.80 \pm 165.90$ & $718.00 \pm 64.20$ & ns \\
\hline reticulocyte $\left(\times 10^{3} / \mathrm{mm}^{3}\right)$ & $194.30 \pm 58.50$ & $177.10 \pm 39.50$ & $296.10 \pm 47.00$ & $253.60 \pm 39.10$ & 0.050 \\
\hline clotting time (s) & $76.00 \pm 27.00$ & $56.00 \pm 21.00$ & $54.00 \pm 15.00$ & $53.00 \pm 14.00$ & ns \\
\hline \multicolumn{6}{|l|}{ Females } \\
\hline hematocrit (\%) & $44.90 \pm 2.24$ & $40.40 \pm 2.31^{*}$ & $43.70 \pm 2.50$ & $45.40 \pm 2.48$ & $\mathrm{~ns}$ \\
\hline hemoglobin (g/dl) & $15.10 \pm 0.73$ & $14.00 \pm 0.70$ & $15.0 \pm 0.80$ & $15.80 \pm 0.81$ & ns \\
\hline red blood cells $\left(\times 10^{6} / \mathrm{mm}^{3}\right)$ & $8.54 \pm 0.49$ & $7.71 \pm 0.45$ & $8.34 \pm 0.31$ & $9.01 \pm 0.56$ & ns \\
\hline white blood cells $\left(\times 10^{3} / \mathrm{mm}^{3}\right)$ & $6.74 \pm 2.27$ & $8.28 \pm 2.32$ & $5.40 \pm 1.06$ & $7.16 \pm 1.32$ & ns \\
\hline \multicolumn{6}{|l|}{ Differential variable $\left(\times 10^{3} / \mathrm{mm}^{3}\right)$} \\
\hline rod neutrophil & $0.0 \pm 0.0$ & $0.04 \pm 0.06$ & $0.0 \pm 0.0$ & $0.0 \pm 0.0$ & ns \\
\hline segmented neutrophil & $1.10 \pm 0.42$ & $2.01 \pm 2.16$ & $0.96 \pm 0.54$ & $1.65 \pm 0.43$ & ns \\
\hline eosinophil & $0.42 \pm 0.09$ & $0.50 \pm 0.24$ & $0.19 \pm 0.13$ & $0.42 \pm 0.43$ & ns \\
\hline basophil & $0.01 \pm 0.02$ & $0.0 \pm 0.0$ & $0.0 \pm 0.0$ & $0.01 \pm 0.03$ & ns \\
\hline limphocyte & $4.98 \pm 2.04$ & $5.56 \pm 1.07$ & $4.15 \pm 0.61$ & $4.76 \pm 1.08$ & ns \\
\hline monocyte & $0.18 \pm 0.15$ & $0.15 \pm 0.13$ & $0.09 \pm 0.05$ & $0.32 \pm 0.14$ & ns \\
\hline blood platelet $\left(\times 10^{3} / \mathrm{mm}^{3}\right)$ & $483.00 \pm 215.00$ & $631.00 \pm 267.00$ & $584.00 \pm 251.00$ & $609.00 \pm 157.00$ & $\mathrm{~ns}$ \\
\hline reticulocyte $\left(\times 10^{3} / \mathrm{mm}^{3}\right)$ & $175.50 \pm 97.30$ & $238.50 \pm 47.90$ & $389.90 \pm 96.00^{*}$ & $359.50 \pm 100.60^{*}$ & 0.005 \\
\hline clotting time (s) & $49.00 \pm 13.00$ & $44.00 \pm 12.00$ & $46.00 \pm 11.00$ & $40.00 \pm 10.00$ & ns \\
\hline
\end{tabular}

Mean values \pm SD for 5 rats.

* Significantly different from the control group at $\mathrm{p} \leq 0.05$.

ns - not significant. 
Table 4. Biochemical parameters from rats exposed to 2-MN for 4 weeks

\begin{tabular}{|c|c|c|c|c|c|}
\hline \multirow{2}{*}{ Parameters } & \multirow{2}{*}{ Controls } & \multicolumn{3}{|c|}{ 2-MN target concentration in inhaled air $\left(\mathrm{mg} / \mathrm{m}^{3}\right)$} & \multirow{2}{*}{$\begin{array}{c}\text { Jonckheere's } \\
\text { trend test } \\
\left(\geq \mathrm{p}_{\mathrm{J}}\right) \\
\end{array}$} \\
\hline & & 2 & 10 & 50 & \\
\hline \multicolumn{6}{|l|}{ Males } \\
\hline alanine aminotransferase $(\mathrm{U} / \mathrm{l})$ & $23.50 \pm 6.80$ & $14.40 \pm 2.10^{*}$ & $19.70 \pm 4.30$ & $16.60 \pm 4.80$ & $\mathrm{~ns}$ \\
\hline alkaline phosphatase (U/l) & $11.50 \pm 9.90$ & $39.00 \pm 15.80^{*}$ & $43.70 \pm 18.90^{*}$ & $43.60 \pm 11.90^{*}$ & 0.010 \\
\hline aspartate aminotransferase (U/l) & $50.50 \pm 9.00$ & $40.60 \pm 10.00$ & $68.90 \pm 32.50$ & $46.00 \pm 14.00$ & $\mathrm{~ns}$ \\
\hline total protein $(\mathrm{g} / \mathrm{dl})$ & $9.00 \pm 0.56$ & $9.30 \pm 0.19$ & $9.40 \pm 0.30$ & $9.40 \pm 0.31$ & ns \\
\hline total cholesterol (mmol/l) & $8.40 \pm 1.40$ & $15.50 \pm 2.20^{* *}$ & $10.20 \pm 6.20$ & $6.60 \pm 0.50^{*}$ & 0.050 \\
\hline$\gamma$-Glutamyltransferase (mU/l) & $0.88 \pm 0.56$ & $0.69 \pm 0.60$ & $0.98 \pm 0.46$ & $1.83 \pm 0.66^{*}$ & 0.010 \\
\hline glucose (mg/dl) & $63.30 \pm 23.70$ & $34.70 \pm 7.80^{*}$ & $35.20 \pm 8.00^{*}$ & $41.00 \pm 11.40$ & ns \\
\hline creatinine (mg/dl) & $1.07 \pm 0.09$ & $1.03 \pm 0.33$ & $0.95 \pm 0.24$ & $0.87 \pm 0.27$ & ns \\
\hline urea $(\mathrm{mg} / \mathrm{dl})$ & $17.80 \pm 3.20$ & $10.60 \pm 2.60^{*}$ & $21.00 \pm 3.00$ & $13.90 \pm 2.50$ & ns \\
\hline sorbitol dehydrogenase (U/l) & $2.40 \pm 1.80$ & $1.40 \pm 1.10$ & $0.83 \pm 0.56$ & $0.58 \pm 0.27$ & 0.010 \\
\hline albumin $(\mathrm{g} / \mathrm{dl})$ & $5.80 \pm 0.30$ & $5.20 \pm 0.40$ & $5.30 \pm 0.20^{*}$ & $4.90 \pm 0.30^{* *}$ & 0.001 \\
\hline sodium $(\mathrm{mmol} / \mathrm{l})$ & $141.00 \pm 1.10$ & $139.00 \pm 1.30^{*}$ & $140.00 \pm 1.70$ & $140 \pm 0.70$ & ns \\
\hline potasium (mmol/l) & $4.40 \pm 0.70$ & $3.80 \pm 0.50$ & $4.40 \pm 0.40$ & $3.9 \pm 0.50$ & $\mathrm{~ns}$ \\
\hline chloride (mmol/l) & $97.00 \pm 3.80$ & $99.00 \pm 3.00$ & $101.00 \pm 2.90$ & $100.00 \pm 2.10$ & ns \\
\hline \multicolumn{6}{|l|}{ Females } \\
\hline alanine aminotransferase (U/l) & $22.10 \pm 9.80$ & $18.70 \pm 7.30$ & $13.80 \pm 4.60$ & $31.50 \pm 3.90$ & ns \\
\hline alkaline phosphatase (U/l) & $53.60 \pm 23.00$ & $42.50 \pm 19.70$ & $28.80 \pm 6.40$ & $35.60 \pm 9.60$ & ns \\
\hline aspartate aminotransferase (U/l) & $103.60 \pm 61.50$ & $67.30 \pm 17.90$ & $73.50 \pm 20.7$ & $91.10 \pm 52.60$ & ns \\
\hline total protein $(\mathrm{g} / \mathrm{dl})$ & $10.00 \pm 0.52$ & $9.60 \pm 0.42$ & $10.30 \pm 0.61$ & $10.00 \pm 0.57$ & ns \\
\hline total cholesterol (mmol/l) & $11.30 \pm 1.30$ & $19.10 \pm 3.80^{* *}$ & $8.60 \pm 2.40$ & $8.20 \pm 2.60$ & 0.020 \\
\hline$\gamma$-Glutamyltransferase (mU/l) & $1.85 \pm 0.89$ & $0.84 \pm 0.72$ & $3.21 \pm 2.13$ & $2.65 \pm 1.15$ & ns \\
\hline glucose (mg/dl) & $31.20 \pm 9.40$ & $42.60 \pm 5.30$ & $41.10 \pm 10.20$ & $48.4 \pm 4.30^{* *}$ & 0.005 \\
\hline creatinine $(\mathrm{mg} / \mathrm{dl})$ & $0.99 \pm 0.27$ & $0.87 \pm 0.17$ & $1.03 \pm 0.33$ & $1.03 \pm 0.33$ & ns \\
\hline urea $(\mathrm{mg} / \mathrm{dl})$ & $16.40 \pm 2.00$ & $20.20 \pm 3.00$ & $21.60 \pm 4.50$ & $24.6 \pm 0.90^{*}$ & 0.001 \\
\hline sorbitol dehydrogenase (U/l) & $2.50 \pm 2.80$ & $0.69 \pm 0.16$ & $1.20 \pm 0.60$ & $2.00 \pm 1.50$ & ns \\
\hline albumin $(\mathrm{g} / \mathrm{dl})$ & $5.80 \pm 0.70$ & $4.90 \pm 0.40^{*}$ & $5.8 \pm 0.40$ & $5.40 \pm 0.60$ & $\mathrm{~ns}$ \\
\hline sodium $(\mathrm{mmol} / \mathrm{l})$ & $137.00 \pm 1.60$ & $138.00 \pm 1.40$ & $137 \pm 3.10$ & $140.0 \pm 2.90$ & 0.050 \\
\hline potasium $(\mathrm{mmol} / \mathrm{l})$ & $4.90 \pm 0.40$ & $4.40 \pm 0.50$ & $4.4 \pm 0.50$ & $4.600 \pm 0.30$ & ns \\
\hline chloride (mmol/l) & $100.00 \pm 1.60$ & $101.00 \pm 3.50$ & $102.00 \pm 1.90$ & $101.000 \pm 1.30$ & ns \\
\hline
\end{tabular}

Mean values SD for 5 rats.

*, ${ }^{*}$ Significantly different from the control group at $\mathrm{p} \leq 0.05$ and $\mathrm{p} \leq 0.01$, respectively.

ns - not significant. 
at $10 \mathrm{mg} / \mathrm{m}^{3}$ produced interstitial infiltrations from mononucleated cells and proteolysis in alveolar and bronchial lumen of three male (3/5) and four female (4/5) rats. Moderate hyperplasia of the peribronchial lymphatic tissue was noted in one male (1/5) and four female (4/5) rats.

Numerous goblet cells were observed in the epithelium of the primary, lobar and segmental bronchi of the animals exposed to $50 \mathrm{mg} / \mathrm{m}^{3}$ of 2-MN; proteinosis with interstitial infiltrations from mononucleated cells was detected in three female (3/5) and three male (3/5) rats. Hyperplasia of peribronchial lymphatic tissue was found to occur in two male (2/5) and three female (3/5) rats.

No pathological changes were noted after patomorphological examinations in the liver, heart, kidneys, spleen, alimentary tract and gonads (both genders), either of the controls or of the rats exposed to 2-MN at $2 \mathrm{mg} / \mathrm{m}^{3}$. Parenchymal steatosis of liver and kidneys was not detected in any of the animals. Hyperplasia of bile ducts was detected in the liver of two male $(2 / 5)$ and three female (3/5) rats exposed to 2-MN at $10 \mathrm{mg} / \mathrm{m}^{3}$ and of all animals exposed to $2-\mathrm{MN}$ at $50 \mathrm{mg} / \mathrm{m}^{3}$. No significant pathologies were noted in the remaining internal organs of the rats exposed to $2-\mathrm{MN}$ at $10 \mathrm{mg} / \mathrm{m}^{3}$ or $50 \mathrm{mg} / \mathrm{m}^{3}$.

\section{DISCUSSION AND CONCLUSIONS}

During the 4-week exposure to 2-MN, the majority of the male rats exposed to various 2-MN concentrations were characterized by similar food intake and similar body mass compared to the male controls (Table 1). The significant reduction in body mass of the male rats exposed to $2-\mathrm{MN}$ at $10 \mathrm{mg} / \mathrm{m}^{3}$ recorded on measurement day 14 is to be regarded as incidental. During the whole exposure period, no statistically significant differences in body mass gain were noted between the 2-MN-exposed female rats and the controls. The observed reduction in body mass of the female rats exposed to 2-MN compared to the controls was due to an evident difference in food intake; during the last two weeks of the experiment (measurement days 21 and 28) food intake of the female rats exposed to 2-MN at $10 \mathrm{mg} / \mathrm{m}^{3}$ or $50 \mathrm{mg} / \mathrm{m}^{3}$ was by about $10-20 \%$ lower compared to the controls.

Our analysis of absolute tissue weights of the 2-MN-exposed animals revealed a significant effect of 2-MN on tissue weight; the effect was more evident and related to more tissues in the female rats. After the 4-week 2-MN exposure, a reduction in the absolute and the relative mass of liver was found to have occurred in all groups of the exposed male rats compared to the controls. The mass values of the remaining internal organs in the male rats exposed to 2-MN generally did not differ from the corresponding values for the controls. The reduction of the mass of spleen and the relative mass of lungs of the male rats exposed to 2-MN at $10 \mathrm{mg} / \mathrm{m}^{3}$ compared to the controls is to be regarded as incidental. An exposureintensity-related reduction in the mass of lungs, liver, spleen, kidneys and heart was evident in the female rats after the 4-week inhalation exposure to 2-MN. When the masses of the internal organs had been converted to relative values specified in terms of grams per 100 grams of rat body mass, it was only the relative mass of spleen which maintained the trend of 2-MN exposure-intensityrelated reduction. The values of the mass of the remaining tissues in 2-MN-exposed animals were in general similar to the corresponding values for the controls. No significant reduction in the relative mass of the majority of the analyzed tissues collected from the 2-MN-exposed female rats compared to the controls results from evidently smaller body mass in all groups of the 2-MN-exposed animals on the last measurement day.

Changed hematological parameters, compared to the control group, were recorded mainly in the male rats. Higher hematocrit values, larger reticulocyte counts, higher hemoglobin concentrations, higher erythrocyte and reticulocyte counts were recorded in the group of the experimental animals. The only significant differences in the female 
rats included significantly higher reticulocyte count, borderline-significant increase in the hemoglobin concentration and higher erythrocyte count. In summary, it seems reasonable to conclude that the hematological changes observed in the 2-MN-exposed rats point to a stimulation of the hematopoietic system.

The majority of the biochemical results show that the studied concentrations of 2-methylnaphthalene do not produce much evident changes in the functioning of the internal organs. Similar aminotransferase activities (elevated aminotransferase activity being a sign of hepatic damage) were noted after the 4-week exposure to 2-methylnaphthalene in experimental rats of both genders relative to control ones $[5,6]$. Increased sorbitol dehydrogenase activity is usually associated with possible hepatic damage whereas, in the present study, lower sorbitol dehydrogenase activity was noted in the male rats; this observation is both surprising and difficult to explain. It is only the increased activity of $\gamma$-glutamyltranferase $(\gamma$-GT) observed in the male rats that may point to cholestasis or early processes leading to hepatic cell damage [7,8]. Some authors suggest that higher $\gamma$-GT activity may indirectly point to stimulation of the cytochrome P-450 enzyme system [9]. The significantly lower cholesterol levels observed in the rats of both genders may indicate possibly disturbed hepatocyte lipids metabolism (e.g., stimulation of hepatocyte microsomal enzymes that transform cholesterol to bile acids). Hypoalbuminemia, that was more evident in the male than in the female rats, might be associated with possible hepatic damage, but also with the observed lower intake of fodder by 2-MN-exposed animals. In a similar way, increased urea concentration in the female rats may point to enhanced protein catabolism, resulting probably from the reduced intake of fodder. The increase of alkaline phosphatase activity observed in male 2-MN-exposed rats was associated probably with incidental low activity of that enzyme in the control rats, the more so that, in 2-MN-exposed female rats, alkaline phosphatase activity was similar as in the controls.
Pathomorphological examinations showed that the 4-week inhalation exposure of rats to 2-MN caused an evident increase in the number of goblet cells in the epithelium of primary, lobar and segmental bronchi. Goblet cells appearing in the epithelium of the lobar bronchi (metaplasia) may point to a weak irritating activity of the studied chemical [10]. The changes in the form of hyperplasia of peribronchial lymphatic tissue accompanied by the production of lymphoepithelium, and interstitial or perivascular infiltrations of varying intensity were also observed, which is quite frequent in conventionally bred rats [11,12]. A 2-MNconcentration-dependent hyperplasia of hepatic bile ducts was also observed. This type of hyperplastic changes is non-specific in its reaction to a number of chemicals, although it may also precede tumors (benign or malicious) from the cells of the intrahepatic bile ducts [12-14].

The results of our study show that repeated exposure of rats to 2-MN produced a number of changes including a) elevated $\gamma$-glutamyltransferase activity, b) stimulation of the erythropoietic system, c) reduced cholesterol concentration, d) increased number of goblet cells found in lobar bronchi, e) non-specific hepatotoxic activity manifested by hyperplasia of bile ducts.

The results of the tests conducted on rats after the 4-week inhalation exposure to 2-MN at various concentrations showed that an evident toxic effect was produced by the chemical at 10 and $50 \mathrm{mg} / \mathrm{m}^{3}$. Thus, it seems reasonable to assume that the 4-week inhalation exposure to 2-MN at $2 \mathrm{mg} / \mathrm{m}^{3}$ represents the lowest-observed-adverse-effect level (LOAEL).

\section{ACKNOWLEDGEMENT}

This research was supported under the statutory activities of the Nofer Insitute of Occupational Medicine (IMP grant 1.4 "Toxicokinetics and toxic effects of exposure to 2-methylnaphtalene in experimental animals"). The authors are grateful to Krzysztof Mader for his excellent technical assistance. 


\section{REFERENCES}

1. Environmental Protection Agency. Toxicological review of 2-methylnaphthalene. EPA 635/R-03/010. Washington: EPA; 2003.

2. Skowroń J.: 2-Methylnaphtalene. Documentation of maximum admissible values for occupational exposure. Podst Met Oceny Srod Pr 2006;22(47):185-204 [in Polish].

3. Dunn OJ. Multiple comparison using rank sums. Technometrics 1964;6:241-52.

4. Jonckheere AR. A distribution-free $k$-sample test against ordered alternatives. Biometrika 1954;41:135-45.

5. Solberg HE, Skrede S, Blomhoff JP. Diagnosis of liver diseases by laboratory results and discriminant analysis. Scand J Clin Lab Invest 1975;35:713-21.

6. Solberg HE, Skrede S, Elgjo K, Blomhoff JP, Gjone E. Classification of liver diseases by clinical chemical laboratory results and cluster analysis. Scand J Clin Lab Invest 1976;36:81-5.

7. Hanke J, Lutz W. Biochemistry, toxicology and laboratory diagnostics of liver. IMP: Łódź; 1986 [in Polish].
8. Burke MD. Liver function: test selection and interpretation of results. Clin Lab Med 2002;22:377-90.

9. Barouki R, Chobert MN, Findori J, Aggerbeck M, NaIpas B, Hanoune J. Ethanol effects in a rat hepatoma cell line: induction of $\gamma$-glutamyltranserase. Hepatology 1983;3(3): 323-29.

10. Witschi H., Nettesheim P.: Mechanisms in Respiratory Toxicology. Vol. I-II. Boca Raton, FL: CRC Press, Inc., 1982.

11. Ribelin W.E., McCoy J.R.: The Pathology of Laboratory Animals. Springfield, Illinois: Ch.C.Thomas Publisher; 1965.

12. Haschek W.M., Rousseaux C.G.: Handbook of Toxicologic Pathology. San Diego: Academic Press; 1991.

13. Greaves P., Faccini J.M.: Rat Histopathology. A Glossary for Use in Toxicity and Carcinogenicity Studie. Amsterdam, New York: Elsevier; 1984.

14. Slott PA, Liu MH, Tavoloni N. Origin, pattern, and mechanism of bile duct proliferation following biliary obstruction in the rat. Gastroenterology 1990;99(2):466-77.

This work is available in Open Access model and licensed under a Creative Commons Attribution-NonCommercial 3.0 Poland License - http://creativecommons.org/ licenses/by-nc/3.0/pl/deed.en. 